\title{
ARTICLE
}

\section{Radiation Transport Calculation Using PHITS Code for Radiation Heat Load and Damage to Superconducting Radioactive Isotope Beam Separator BigRIPS at RIKEN}

\author{
Tetsuya OHNISHI*, Kensuke KUSAKA and Toshiyuki KUBO
}

RIKEN Nishina Center, RIKEN, Hirosawa 2-1, Wako, Saitama, 351-0198, Japan

\begin{abstract}
The BigRIPS separator is the superconducting in-flight radioactive isotope (RI) beam separator at RIKEN Nishina Center. In the RI production process with an intense primary beam, the radiation heat load and damage are critical issues for the BigRIPS separator. To estimate these quantities, the radiation transport calculation was performed by using the PHITS code. The PHITS code reproduces the measured heat load data within a factor of 1.5.
\end{abstract}

KEYWORDS: radiation transport calculation, PHITS code, heavy ion beams, ${ }^{48}$ Ca beam, radiation heat load, radiation damage, in-flight $\mathrm{RI}$ beam separator, superconducting magnet

\section{Introduction}

In 2007, a new-generation radioactive isotope (RI) beam facility called "RI beam factory" (RIBF) ${ }^{1)}$ has started its operation at RIKEN Nishina Center. At RIBF, the BigRIPS, ${ }^{2)}$ which is a new-generation superconducting in-flight RI beam separator, is used to produce variety of RI beams by the projectile fragmentation of various heavy ions, or in-flight fission of a ${ }^{238} \mathrm{U}$ beam. In this facility, heavy ions up to uranium can be accelerated to $345 \mathrm{MeV} /$ nucleon. The goal intensity at RIBF is as high as $6.2 \times 10^{12}$ particles $/ \mathrm{sec}$, and the maximum beam power reaches to $83 \mathrm{~kW}$ in the case of the uranium beam.

Figure 1 shows a schematic layout of the BigRIPS separator, which consists of 14 superconducting triplet quadrupoles (STQ1-STQ14) ${ }^{3-5)}$ and 6 room-temperature dipoles (D1-D6). There are seven foci (F1-F7) in the beam line. The BigRIPS separator is characterized by two-stage separator scheme. ${ }^{2}$ The first stage from the production target at the F0 focus to the F2 focus is used for the production and separation of RI beams, and the second stage from the F3 focus to the F7 focus is employed to identify RI beams in an event-by-event mode.

A rotating target system $^{6)}$ is installed at F0, and a high-power beam dump ${ }^{7)}$ is placed inside the gap of the first dipole D1 as well as at the exit side of D1. The beam dump at the exit of D1 is a movable one in order to stop a primary beam at different position depending on the RI beam production condition.

In the production process of RI beams, high-energy light charged particles and neutrons are produced at the production target and the beam dump. These particles hit the beam line devices, especially STQ1 just after the production target and STQ2 at the downstream of the beam dump. These STQs are cooled by the liquid helium cryogenic plant ${ }^{3)}$ which has a total cooling capacity of about $500 \mathrm{~W}$ at $4 \mathrm{~K}$ and a extra cooling capacity of about $300 \mathrm{~W}$ at $4 \mathrm{~K}$. The total radiation heat load to the cryostats of STQ1 and STQ2 makes serious problem on the operation of the cryogenic plant, if it exceeds $300 \mathrm{~W}$. The heat load density at the superconducting coil of the STQ is also important in order to

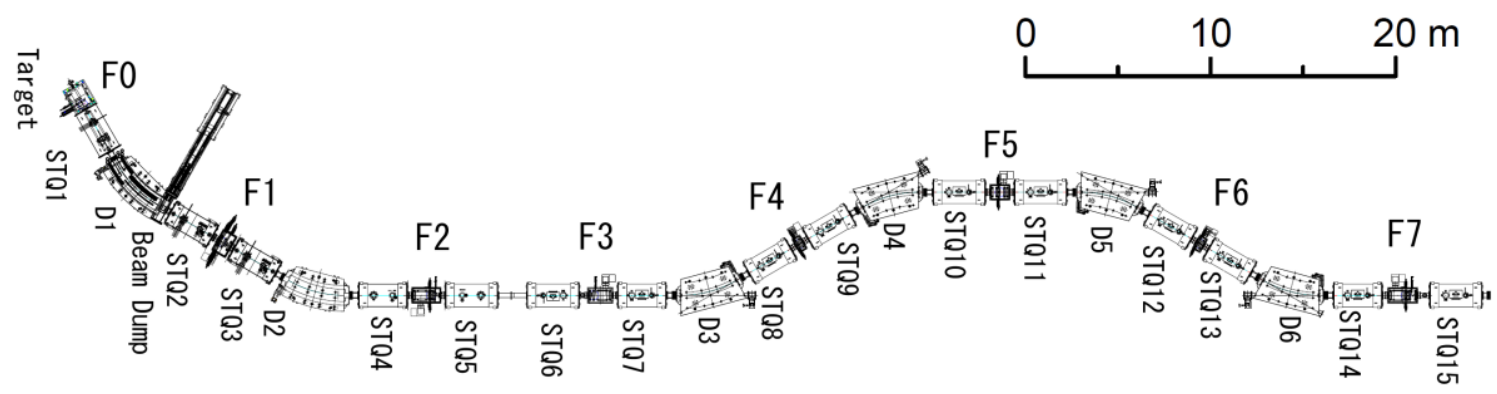

Fig. 1 Schematic layout of the BigRIPS separator. The STQn and Dn denote the superconducting quadrupole triplets and the room-temperature dipoles, respectively. Fn indicates the focus.

*Corresponding author, E-mail: oonishi@ ribf.riken.jp

(C) 2011 Atomic Energy Society of Japan, All Rights Reserved. 
keep the coil in the superconductive state. The radiation damage is one of the critical issues, because it limits the lifetime of the beam line devices.

The radiation heat load to the cryostat containing STQ1 was first observed with a ${ }^{48} \mathrm{Ca}^{20+}$ beam at $345 \mathrm{MeV} /$ nucleon in December 2008. The setting of the BigRIPS separator was set to the production of the neon isotopes, ${ }^{31} \mathrm{Ne}$ and ${ }^{32} \mathrm{Ne}$. This heat load was caused by a large number of the high-energy light charged particles and neutrons produced at the production target.

In May and June 2010, a series of experiments on oxygen and aluminum isotopes was performed with an intense ${ }^{48} \mathrm{Ca}^{20+}$ beam at $345 \mathrm{MeV} /$ nucleon. The radiation heat load to the STQ2 cryostat as well as the STQ1 cryostat was observed during the aluminum isotope experiment. In this case, the source of the radiation heat load to the STQ2 cryostat was the charged particles and the neutrons from the movable beam dump, because it was set to the location in the beam bore tube of the STQ2 cryostat.

A radiation transport calculation, which can transport heavy ions, is required to estimate the radiation heat load and damage caused in the RI beam production. PHITS (multi-purpose Monte Carlo Particle and Heavy Ion Transport code System ${ }^{8)}$ is one of the calculation code which includes reaction processes of heavy ions as well as light particles.

In this paper, we report details of the radiation transport calculation on the radiation heat load and damage using the PHITS code and the comparison with the experimental data.

\section{Calculation Model}

The radiation transport calculation was performed by using the PHITS code. ${ }^{8)}$ In the PHITS code, the collisions of light particles and heavy ions with materials are calculated

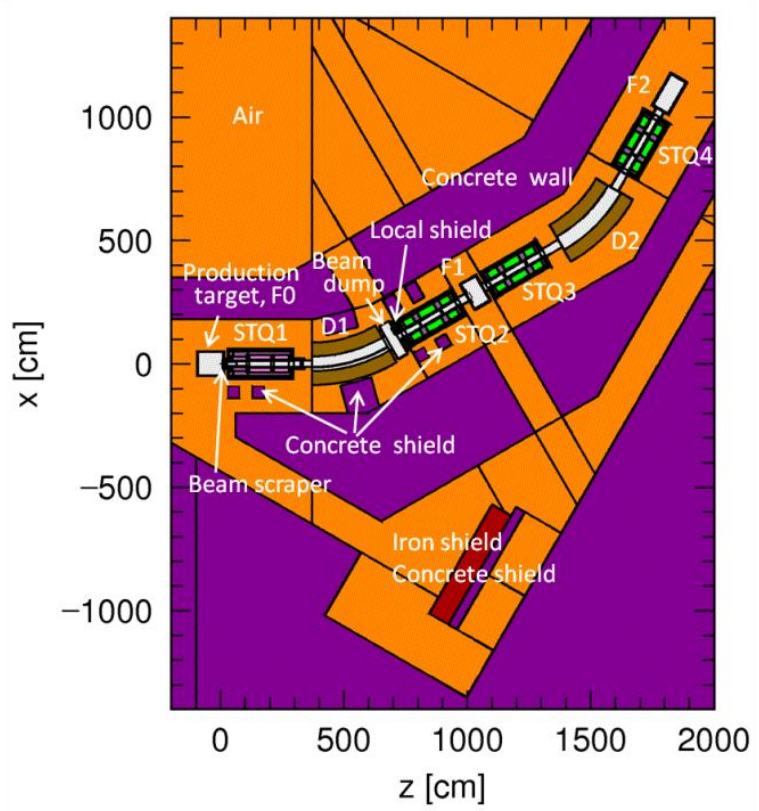

Fig. 2 Schematic layout of the calculation model of the BigRIPS separator

by using the JAM and JQMD models, ${ }^{8)}$ respectively. Evapo- ration and fission processes of heavy ions are also included. The PHITS code employs the cross sections from evaluated nuclear data libraries for low energy protons and neutrons. ${ }^{8)}$ To get enough event sources and a fast computation, the calculation was made at RIKEN Integrated Cluster of Clusters (RICC) facility, which has a 107 TFLOPS performance and 8,192 CPUs.

Figure 2 shows the schematic drawing of the beam line of the BigRIPS separator from the production target to the F2 focus in the calculation model. Beam line devices in this model were the production target, the magnets, the beam dump, the vacuum chambers, the concrete shields around the magnet, and the concrete walls. A prototype beam scraper ${ }^{9)}$ was installed at the entrance of STQ1 inside the beam bore tube in order to reduce the heat load to STQ1. The beam scraper was a $126 \mathrm{~mm}$ long copper block with the collimation hole which was $36 \mathrm{~mm}$ in a diameter. In addition, a local shield between the beam dump and STQ2 was placed to prevent STQ2 from particles produced at the beam dump. The local shield consists of tungsten and iron shields. The structure of the beam line devices was modeled in a three-dimensional way with their constituent materials.

In the calculation, a sharp edge shape was used for the magnetic field of the quadrupole and the dipole. The correction of the effective length was applied to the magnetic field of the quadrupole. The strength of the magnetic field was set to the experimental values.

The PHITS code transports not only primary beam and fragments produced in the target, but also neutrons, protons and light charged particles produced along the beam line of the BigRIPS separator. As a result of the transport calculation, we obtain the estimation of the radiation heat load and damage to the beam line devices.

\section{Measurement and Comparison with PHITS Calculation}

\section{Measurement of Radiation Heat Load}

In 2008 and 2010, an intense ${ }^{48} \mathrm{Ca}^{20+}$ beam at $345 \mathrm{MeV} /$ nucleon was employed to produce RI beams. The maximum beam intensity was reached to about $4 \mu \mathrm{A}$. The heat load to the STQ1 cryostat was clearly observed by comparing the heater power of STQ1 with and without the beam irradiation. The heater power of the cryostat was controlled to keep the liquid helium level in the cryostat, so that the heat load was compensated by the heater power. To deduce the heat load from the measurement of the heater power, the heat load analysis of STQ was performed. ${ }^{10)}$

Table 1 shows the summary of the experimental settings performed in 2008 and 2010, and the measured heat load to the STQ1 cryostat. The settings used in the ${ }^{31} \mathrm{Ne},{ }^{32} \mathrm{Ne},{ }^{24} \mathrm{O}$, and ${ }^{33} \mathrm{Al}$ isotope experiments are named as Setting I, II, III, and IV, respectively. The following paragraphs describe more detail of the measurements.

In 2008, the ${ }^{31} \mathrm{Ne}$ and ${ }^{32} \mathrm{Ne}$ isotopes were produced with $15 \mathrm{~mm}$ and $20 \mathrm{~mm}$ thick Be targets with a ${ }^{48} \mathrm{Ca}^{20+}$ beam at $345 \mathrm{MeV} /$ nucleon, respectively. The beam current was 
Table 1 Experimental condition and measured heat load to the STQ1 cryostat

\begin{tabular}{cccccc}
\hline Setting & $\begin{array}{c}\text { Target } \\
\text { isotope }\end{array}$ & $\begin{array}{c}\mathrm{B}^{*} \\
{[\mathrm{Tm}]}\end{array}$ & $\begin{array}{c}\text { Be target } \\
\text { thickness } \\
{[\mathrm{mm}]}\end{array}$ & $\begin{array}{c}\text { Average } \\
\text { current } \\
{[\mu \mathrm{A}]}\end{array}$ & $\begin{array}{c}\text { Heat } \\
\text { load } \\
{[\mathrm{W}]}\end{array}$ \\
\hline $\mathrm{I}$ & ${ }^{31} \mathrm{Ne}$ & 8.2 & 15 & 0.53 & 11.9 \\
$\mathrm{II}$ & ${ }^{32} \mathrm{Ne}$ & 8.4 & 20 & 2.25 & 42.6 \\
$\mathrm{III}^{\dagger}$ & ${ }^{24} \mathrm{O}$ & 8.1 & 15 & 3.5 & 32.7 \\
$\mathrm{IV}^{\dagger}$ & ${ }^{33} \mathrm{Al}$ & 7.0 & 10 & 3.7 & 26.8 \\
\hline
\end{tabular}

"Magnetic rigidity used for the optics from the F0 focus to the

F1 focus in the BigRIPS separator.

${ }^{\dagger}$ The beam scraper was installed inside STQ1.

measured by a fast current transformer ${ }^{11)}$ (FCT) which was installed at upstream of the target chamber. The FCT reading was calibrated with a Faraday cup reading. During the experiments, the heater power decreased clearly when the beam bombarded the target. Taking the average of the heater power and the beam current, we evaluate the radiation heat load caused by the beam, which is shown in Table 1 .

The ${ }^{24} \mathrm{O}$ isotope experiment was performed with a ${ }^{48} \mathrm{Ca}^{20+}$ beam at $345 \mathrm{MeV} /$ nucleon in May 2010. The production target was $15 \mathrm{~mm}$ thick Be target. The beam scraper, which is described in Section II, was installed at the entrance of STQ1. The beam intensity was monitored by using the phase probe at the exit of the accelerator calibrated with a Faraday cup reading, because the FCT beam monitor was not available in this beam time. The heat load was estimated in the same way as in 2008, and it was $32.7 \mathrm{~W}$ with the beam intensity of $3.5 \mu \mathrm{A}$.

In June 2010 , the ${ }^{33} \mathrm{Al}$ isotope experiment was also performed using $10 \mathrm{~mm}$ thick Be target with the same Ca beam as the ${ }^{24} \mathrm{O}$ isotope experiment. In this experiment, the movable beam dump at the exit of D1 was set to the position within the beam bore tube of the STQ2 cryostat, because the stop position of the primary beam was $67 \mathrm{~mm}$ apart from the central beam axes. The beam dump became the main source of neutrons and light charged particles which directly hit STQ2. As a result, the heat load to the STQ2 cryostat was first observed about $13 \mathrm{~W}$, while the heat load to the STQ1 cryostat was about $26.8 \mathrm{~W}$. The beam intensity was about $3.7 \mu \mathrm{A}$ monitored in the same way as the ${ }^{24} \mathrm{O}$ isotope experiment.

The systematic error of the measured heat load is estimated to be about $30 \%$. The error comes from the averaging of the heater power and the determination of the beam intensity. a) Neutron Horizontal plane

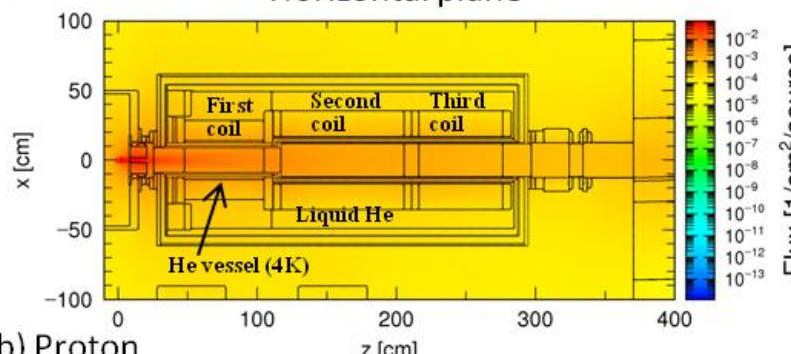

b) Proton

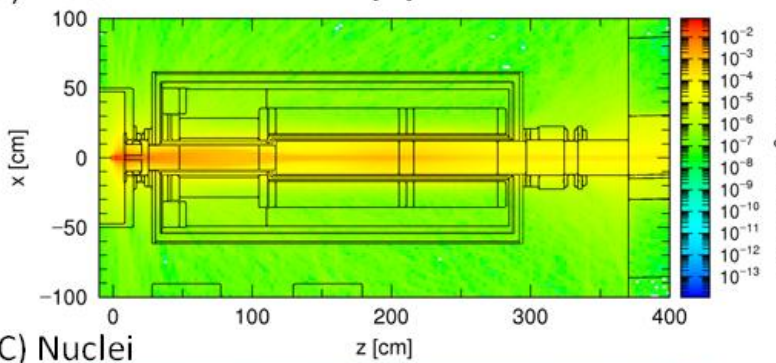

C) Nuclei

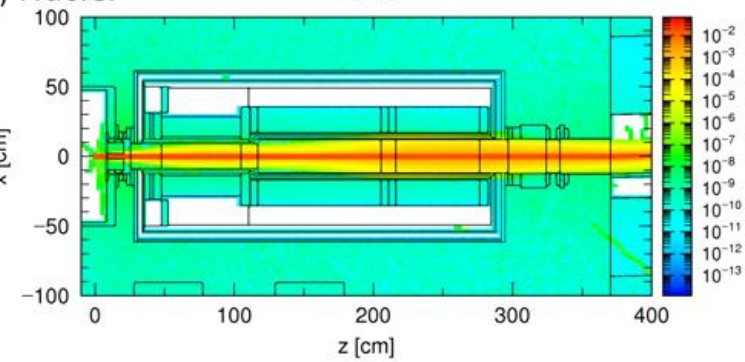

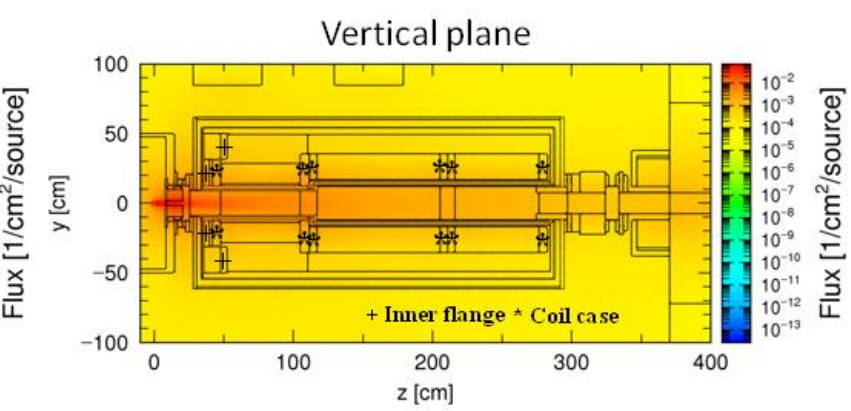
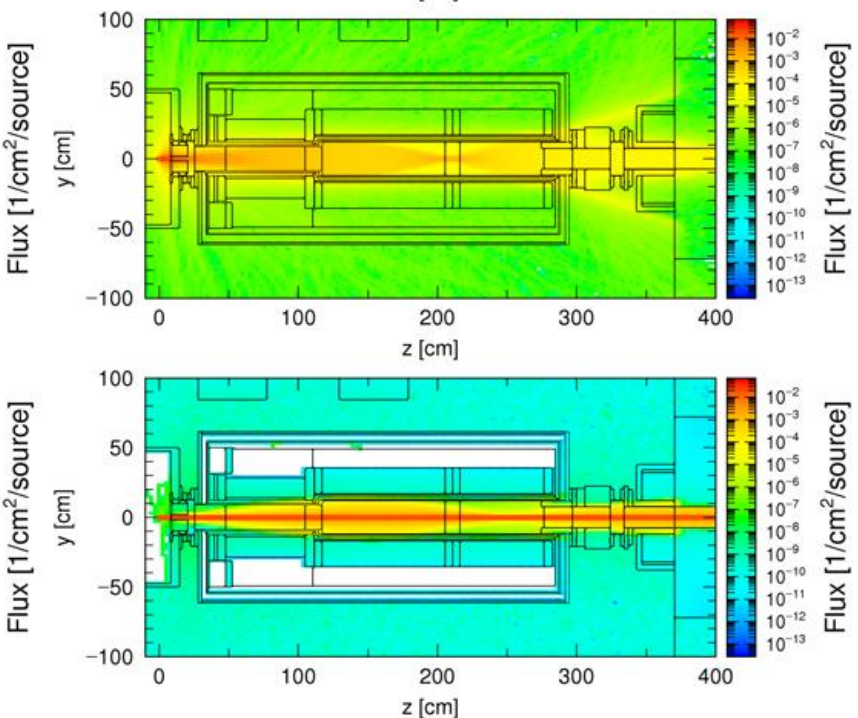

Fig. 3 Calculated flux intensity of particle around STQ1 in Setting III. The flux intensities of a) neutrons, b) protons and c) nuclei including primary beam are plotted in units of $\left[1 / \mathrm{cm}^{2} /\right.$ source]. Here, the normalization factor "per source" means "caused by one nucleus of primary ${ }^{48} \mathrm{Ca}$ beam". Left and right panels show the flux intensity in the horizontal and vertical cross sections, respectively. 


\section{Radiation Heat Load Estimation Using PHITS}

We performed the radiation transport calculation of Setting I, II, III, and IV by using the PHITS code, and estimated the radiation heat load. The experimental values were applied to the production target thickness, the shape of the primary beam, and the magnetic field settings. Concerning the shape of the primary beam, a Gaussian shape was applied to the position and angular distribution of the primary beam, and the width of the distribution was obtained from the experimental data. The calculation was performed by generating about a million event sources.

Figure 3 shows the calculated distribution of the flux intensities of neutrons, protons, and nuclei including primary beam around STQ1 in Setting III (Table 1). Neutrons are produced at the target, and they pass through STQ1 mainly in the forward direction. Protons produced at the target are transported inside STQ1, and hit the inner duct of STQ1 in both horizontal and vertical directions due to its different optics from objected isotope and its large spreads in size and angle.

The heat load distribution around STQ1 calculated in Setting III is shown in Fig. 4. In Fig. 4, the heat load mainly distributes at the inner duct of STQ1. This is supposed to be caused by protons, compared with the flux shown in Fig. 3. The heat load around the first coil is larger than around other coils, because the first coil is close to the production target.

Table 2 shows the calculated heat load to the STQ1 cryostat with the experimental settings listed in Table 1. In addition, we calculated the heat load with a setting which is the same as Setting I except for using the beam scraper, named as Setting I'. All results in Table 2 correspond to the beam intensity of $20 \mu \mathrm{A}$. Total heat loads are calculated by integrating the heat density over each part of the STQ1 component. The statics error of the calculated total heat load
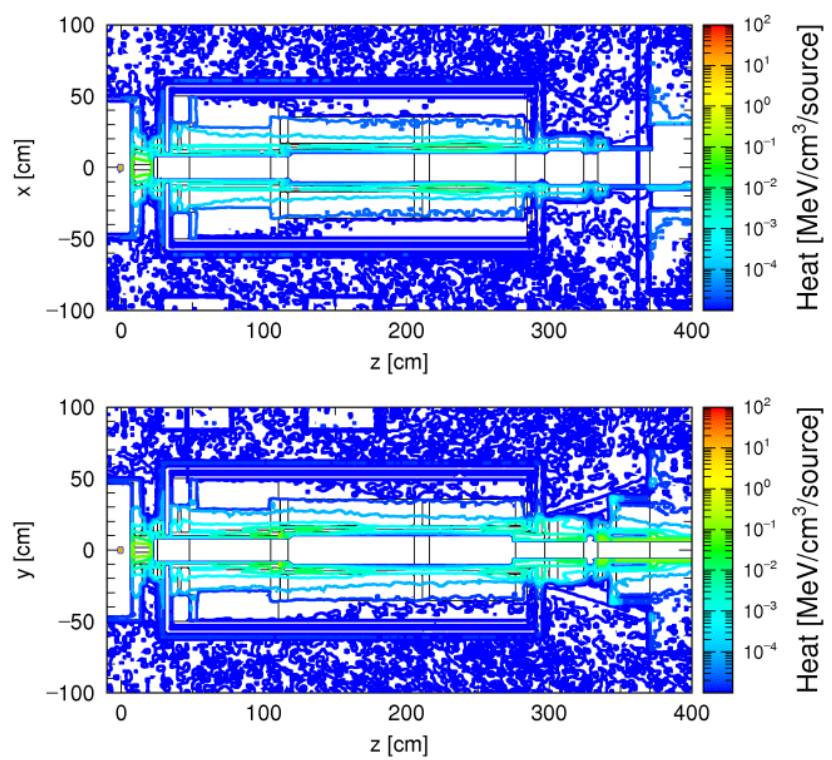

Fig. 4 Heat load distribution around STQ1 in Setting III. Upper and lower panels show the distribution in the horizontal and vertical cross section, respectively. The normalization factor "per source" is the same as in Fig. 3.
Table 2 Estimated heat load by PHITS calculation

\begin{tabular}{cccccc}
\hline Setting & I & I $^{*}$ & II & III $^{*}$ & IV $^{*}$ \\
\hline Liquid He & 22.1 & 13.1 & 28.0 & 12.8 & 9.01 \\
First coil & 23.5 & 9.40 & 28.7 & 9.14 & 6.58 \\
Second coil & 11.1 & 7.58 & 13.7 & 7.33 & 5.35 \\
Third coil & 4.31 & 4.01 & 5.24 & 3.88 & 2.89 \\
First coil case & 121 & 47.6 & 151 & 45.9 & 31.4 \\
Second coil case & 27.3 & 18.5 & 33.5 & 17.9 & 12.9 \\
Third coil case & 16.3 & 14.6 & 20.0 & 13.8 & 9.30 \\
Inner flanges & 18.6 & 8.40 & 23.0 & 8.14 & 5.80 \\
He vessel & 208 & 147 & 263 & 143 & 102 \\
\hline Total 4K load & 452 & 271 & 567 & 262 & 186
\end{tabular}

All numbers are given in unit of $\mathrm{W}$. The results are calculated with the beam intensity of $20 \mu \mathrm{A}$.

* The beam scraper is included inside STQ1.

with Monte Carlo method is estimated to be less than $0.01 \%$.

Scaling with the experimental beam intensity, total $4 \mathrm{~K}$ heat load to the STQ1 cryostat was estimated to be 12.0, 63.8, 45.9, and 34.4 W for Setting I, II, III, and IV, respectively. The estimated results are about 1.3 - 1.5 times larger than the measured heat loads in Table 1 except Setting I.

To evaluate the effect of the beam scraper, we compare the calculated results of Setting I and I'. The beam scraper reduces the total $4 \mathrm{~K}$ heat load about $40 \%$. This effect is confirmed using the measured heat load in Setting I and III, because the target thickness and magnetic rigidity in Setting III is almost the same as in Setting I. Scaling the measured heat load, the reduction using the beam scraper is about $58 \%$. It almost agrees with the calculation.

Concerning STQ2, the heat load to the STQ2 cryostat was estimated in Setting IV. The estimated heat load was $10.5 \mathrm{~W}$, while the measured heat load was $13 \mathrm{~W}$. The PHITS code also reproduces the heat load to the STQ2 cryostat.

\section{Estimation of Absorbed Dose at First Coil of STQ1}

To estimate the radiation damage, the peak value of the absorbed dose at the first coil of STQ1 was obtained from the calculated heat load distribution.

Results of the peak of heat load density and absorbed dose are summarized in Table 3. The results are calculated with the beam intensity of $20 \mu \mathrm{A}$. The superconducting coil of the STQ consists of NbTi and copper, and its radiation tolerance is about $10^{8} \mathrm{~Gy}$. Using the calculated value in Setting III, the lifetime of the first coil of STQ1 is estimated about 30 years

Table 3 Estimated peak dose at the first coil of STQ1

\begin{tabular}{ccc}
\hline Setting & $\begin{array}{c}\text { Peak heat load density } \\
{\left[\mathrm{mW} / \mathrm{cm}^{3}\right]}\end{array}$ & $\begin{array}{c}\text { Peak absorbed dose } \\
{[\mathrm{Gy} / \mathrm{sec}]}\end{array}$ \\
\hline I & 9.9 & 1.1 \\
II & 13 & 1.5 \\
III & 3.0 & 0.30 \\
IV & 2.2 & 0.24 \\
\hline
\end{tabular}

The results are calculated with the beam intensity of $20 \mu \mathrm{A}$. 
with the beam intensity of $20 \mu \mathrm{A}$ and the beam time period per year of $10^{7}$ seconds. The epoxy resin insulation, of which its radiation tolerance is about $10^{7} \mathrm{~Gy}$, is also used in the coil of the STQ. If the coil consists of only epoxy, the calculated peak heat load density and absorbed dose are $0.75 \mathrm{~mW} / \mathrm{cm}^{3}$ and $0.61 \mathrm{~Gy} / \mathrm{sec}$ in Setting III, respectively. The estimated lifetime of the epoxy resin insulation is about 2 years with the same beam intensity and period condition used in the case of the NbTi and copper coil.

\section{Summary}

In summary, we have performed the radiation transport calculation for the radiation heat load and damage to the BigRIPS separator by using the PHITS code. We compared the calculated heat load to STQs with the measured values in several experimental settings with an intense ${ }^{48} \mathrm{Ca}^{20+}$ beam at $345 \mathrm{MeV} /$ nucleon. The PHITS code reproduces the measured heat load within a factor of 1.5. The results will be used for the final design of the beam scraper at STQ1 and the optimization of the local shield of the beam line devices.

\section{References}

1) Y. Yano, "The RIKEN RI Beam Factory Project: A status report," Nucl. Instr. Meth. Phys. Res., B261, 1009-1013 (2007).
2) T. Kubo, "In-flight RI beam separator BigRIPS at RIKEN and elsewhere in Japan," Nucl. Instr. Meth. Phys. Res., B204, 97-113 (2003).

3) T. Kubo et al., "Status and overview of superconducting radioactive isotope beam separator BigRIPS at RIKEN," IEEE Trans. Appl. Superconductivity, Vol. 17, 1069-1077 (2007).

4) K. Kusaka et al., "An Air-core Type Superconducting Quadrupole Triplet for the BigRIPS Separator at RIKEN," IEEE Trans. Appl. Superconductivity, 18, 240-243 (2008).

5) K. Kusaka et al., "Prototype of Superferric Quadrupole Magnets for the BigRIPS Separator at RIKEN," IEEE Trans. Appl. Superconductivity, 14, 310-315 (2004).

6) A. Yoshida et al., "Status and overview of production target for BigRIPS separator at RIKEN," Nucl. Instr. Meth. Phys. Res., A590, 204-212 (2008).

7) N. Fukuda et al., "High-power beam dump system for BigRIPS," RIKEN Accel. Prog. Rep., 41, 119-120 (2008).

8) K. Niita et al., "PHITS a particle and heavy ion transport code system," Radiat. Meas., 41, 1080-1090 (2006).

9) A. Yoshida et al., "Upgrading the BigRIPS target chamber," RIKEN Accel. Prog. Rep., 43, 153-154 (2010).

10) K. Kusaka et al., "Radiation Heat Load to Superconducting Quadrupoles for BigRIPS In-flight Separator at RIKEN," IEEE Trans. Appl. Superconductivity, in press.

11) http://www.bergoz.com 